\title{
Improvement of Immune System Analyzed through Various Cluster Techniques - A Survey
}

\author{
M Veronica Arokia Mary, K R Sekar, R Manikandan, K S Ravichandran
}

\section{M veronica Arokia Mary, K R Sekar, R Manikandan, K S Ravichandran}

School of Computing, SASTRA Deemed University, Thanjavur, Tamil Nadu, INDIA.

Correspondence

M veronica Arokia Mary

School of Computing, SASTRA Deemed University, Thanjavur, Tamil Nadu, INDIA.

Phone no: 9952445079

E-mail: veronmariadass@gmail.com

History

- Submission Date: 22-12-2017;

- Review completed: 02-01-2018;

- Accepted Date: 08-02-2018

DOI : 10.5530/pj.2018.2.45

Article Available online

http://www.phcogj.com/v10/i2

\section{Copyright}

(C) 2018 Phcog.Net. This is an openaccess article distributed under the terms of the Creative Commons Attribution 4.0 International license.

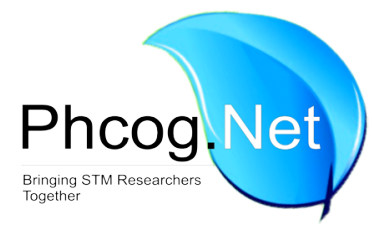

\begin{abstract}
The world health organization report says the immunity deficiency is the biggest problem in the all over the world. Immune system can be improved through many fruits, especially grape is inevitable fruit that provides high neutrinos. The grape contains phytonutrients, Phenols and Poly phenols in rich amount. It is used for constipation, indigestion, fatigueless, kidney disorder, Macular degeneration and cataracts. The grape seed reduces swelling, Eye disease caused by diabetics and acting as antioxidants. In the research article, plenty number of classifiers were used to identify good type of grapes that provides the above said. The methodology applied in the work were Ada Boost and Machine learning Meta algorithm. The above areas were reviewed to the core.
\end{abstract}

Key words: Ada boost classifier, Grape detection, Color components, Segmentation techniques, Immune systems, Phytonutrients.

\section{INTRODUCTION}

The grape provides high protein, vitamins and minerals to the body for the improvement of immune system. It is one price worthy fruit that enhances fiber, hydrate the body gives healthy carbs acting as nan antioxidant, provides moderate proteins and myricetin. The flavanoids and quercetin will reduce the damage to the body caused by free radicals.

In the grape has got essential minerals like potassium, calcium, magnesium, phosphors and sodium are the good electrolids. The Vitamins like K, A, C and B6, moreover thiamine, riboflavin, niacin and folate provides good immune system to the human body. A grape cures disease like Asthma, Bone weakness, Heart disease, Migraine problems, reduces blood cholesterol, acting as an antibacterial, avoids breast cancer, reduces risk of Alzheimer and improves immunity to the body.

Grapes are one of the common fruit in the world and that can be growing. ${ }^{1}$ in the cluster of 15 to $300 \mathrm{~m}$. According to food and agriculture organization, the grape cultivation is processed needs a lot of people to do the work and its lead to more time-consuming, and labor- intensive. ${ }^{2}$ process for cultivation and harvesting. The method is used for automatic harvesting is based on artificial vision, computer vision. The Vineyard images ${ }^{3}$ can be further divided into RGB color components. Using color images, the features can be extracted and preprocessing with ${ }^{4}$ similar color component methods. RGB images is used for capturing the grape bunches at night under the light conditions. The conventional RGB color images can be transformed the different color spaces with val- ues of HSV, YIQ, with intensity values of a particular images. To detect a fruit color skins. ${ }^{5,6}$ the pixel color, intensity distance can be measured by the linear color model. To avoid an illumination variability at night time, the segmentation procedure is used. The segmentation technique for each pixel images based on applying Bayesian Discrimination models. The results were stored in the LUT (Look up Table) to perform a segmentation process in faster manner. In cluster detection can be carried out with the various classifiers and segmentation algorithm was proposed here. ${ }^{7,8}$ Support Vector Machines are supervised learning that can be analyzed data used for classification. ${ }^{9,10}$

\section{Vineyard Detection by Various Boosting and Classifier Methods}

\section{Adaboost classifier}

AdaBoost is also known as an Adaptive Boosting classifier. It is a method of machine learning algorithm by a set of training with a boosted classifier. The Boosting algorithm developed for binary classification, for solving the classification problems, a modified boosting method based on AdaBoost algorithm.the combination methods based on classifier based on either ensemble or committee classifiers. ${ }^{11,12}$

Two sets of an experiment can be carried out for new boosting algorithm first one is comparing boosting and bagging. This bagging method is also known as Bootstrap Aggregating which is a machine learning ensemble with Adaboost algorithm which is used to design for improving a stability and accuracy. ${ }^{13}$ The 

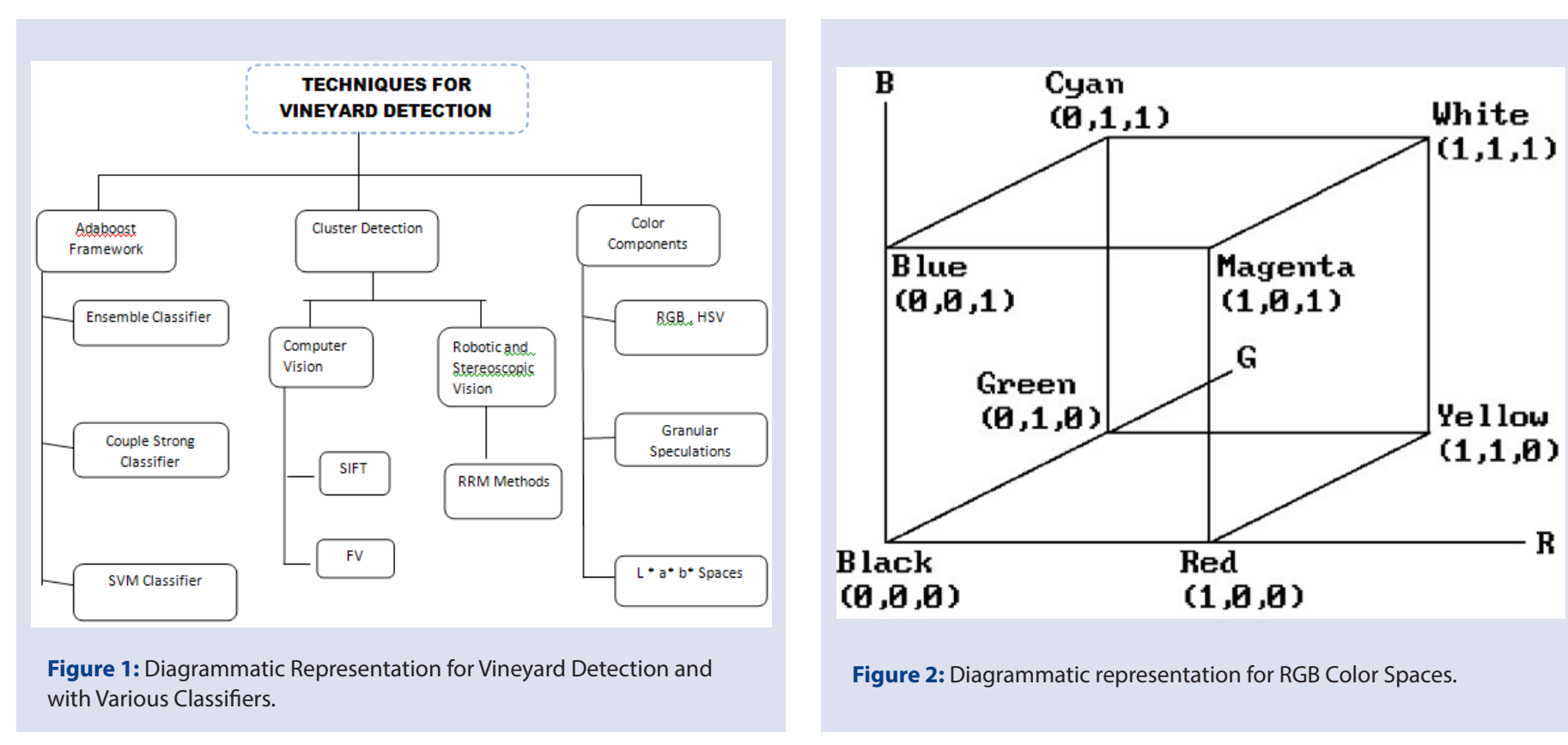

Figure 1: Diagrammatic Representation for Vineyard Detection and with Various Classifiers.

Figure 2: Diagrammatic representation for RGB Color Spaces.

Table 1: Color Components of both Linear and Non-Linear Transformations.

\begin{tabular}{ccccc}
\hline Color models & $\begin{array}{c}\text { Linear transformations } \\
\text { values }\end{array}$ & $\begin{array}{c}\text { Non-linear } \\
\text { transformations values }\end{array}$ & $\begin{array}{c}\text { Image pixel range in } \\
\text { degrees }\end{array}$ & $\begin{array}{c}\text { Image segmentation } \\
\text { in } \%\end{array}$ \\
\hline YUV & 0.617 & 0.715 & 90 degrees & $65 \%$ \\
HSV & 0.174 & 1.116 & 45 degrees & $47 \%$ \\
HIS & 0.299 & 0.177 & 60 degrees & $82 \%$ \\
YUI & 0.200 & 0.114 & 30 degrees & $68 \%$ \\
CIE & 0.000 & 0.874 & 110 degrees & $87 \%$ \\
\hline
\end{tabular}

Table 2: Representations of Various Color Spaces Methods.

\begin{tabular}{|c|c|c|}
\hline COLOR SPACES & ADVANTAGES & DISADVANTAGES \\
\hline RGB & It provides a complete display for monitoring & $\begin{array}{l}\text { The color image processing can be damaged because of the high } \\
\text { correlation values }\end{array}$ \\
\hline YIQ & The color information varied within the detected images & $\begin{array}{c}\text { The values in YIQ which cannot be less prediction based on the RGB } \\
\text { values }\end{array}$ \\
\hline YUV & $\begin{array}{c}\text { It is used for enhancing the rays obtained from the light } \\
\text { illumination of the images. }\end{array}$ & $\begin{array}{l}\text { Correlation values can be differing from the linear } \\
\text { Transformation, when it cannot be predicted as high as RGB. }\end{array}$ \\
\hline HIS & $\begin{array}{l}\text { The value of the color ranges for human eye perceptions the } \\
\text { illumination can perform for HIS values }\end{array}$ & Independent colors compared to other color values \\
\hline CIE & $\begin{array}{l}\text { The value of CIE intensity information can } \\
\text { Independent and it used for easier detections, }\end{array}$ & Lesser dependent image values. \\
\hline
\end{tabular}

boosting algorithm was developed with new versions to be denoted as Adaboost.M1, AdaboostM2.

Bagging method is used for combining the multiple copies of learning datasets. The performance of the learning algorithm combines AdaBoost with the nearest neighbor classifiers. Strong classifiers are used in the AdaBoost framework where weak classifiers are connected in either sequential or parallel in it. ${ }^{14}$
To improve the weak classifier the algorithm can be used as CSCsCoupled Strong Classifiers. The CSCs concept is based on the following as ACCFs (Aggregated Channel Comparisons Features) and LDCFs (Locally De- Correlated Channel Features). ${ }^{15,16}$ Pedestrian Detection is a method of detecting any intelligent video surveillance systems, by using this application CSCs in AdaBoost can be implemented. This detection which improves the performances about $8 \%$ of miss rate and means rate to improve the further enhancement of AdaBoost framework. ${ }^{17,18}$ 


\section{Various Vision Techniques for Automatic Detection for Different Plants}

In this survey the automatic detection and visions for various plants are discussed here. Now a day's Automatic detection is still a challenge for a harvesting the robot. A major challenge for a robotic harvesting can be explained in three issues: fruit images recognition by $\mathrm{RGB}^{19,20}$ hand-eye images for coordination.and end-effectors. computer vision can be used for the analysis and understanding the useful information for either a single or sequence of images. The precision agriculture plays a vital role in the growth of the heading stage of the wheat plants. In this paper the automatic observation for wheat heading stage is based on computer visions. Heading stage is the stage when the panicle is fully visible so that heading can be low spatial resolution images it was observed by SIFT (Scale Invariant Feature Transform) and FV (Fisher Vector). ${ }^{23,24}$

Stereoscopic vision stereo- meaning "solid", "appearance of sight". Automatic fruit detection by using a laser-based computer vision. In this paper infrared laser is used for finding ranges and reflectance images in the fruit harvesting. In stereo vision main difficulties that can be focused in this paper is stereo matching and cross correlation functions. ${ }^{25}$

The circle shape of the apples can be detected based on RRM (Random Ring Method), the radius of the apple can be determined by the center of the apple. Segmentation of the apples based on recognizing with the certain range of (30-360) degree pixels during shooting distances from the camera.

Another technique of fruit detection by recognition for robotic harvest based on feature image fusions. Tomato was collected and performed by using image acquisition method by taking 200 samples of images.

These images can be extracted by using color feature extractions (RGB, HSV, $\mathrm{L}^{*}, \mathrm{a}^{*}, \mathrm{~b}^{*}$ ) color components done by wavelet transform by an orthogonal set of images. ${ }^{26,27}$

\section{Color Components of Images with Natural Environment}

The color segmentation is play an important role in detecting and partitioning the digital images into various multiple segments. Each Segmentation was assigned with different pixel images.

The color images can be segmented towards 4 segmentation techniques with a color features, in this paper introduces the segmentation process for developing fruit images to provide the histogram values based on predicting with a neural network. ${ }^{28}$

In the reference ${ }^{29}$ surveyed segmentation algorithms based on threshold and to perform the values on segment using uniformity and shape measures.

The Color for detecting the various color spaces such as R(red), G(green), and $\mathrm{B}$ (blue) which is used for finding the grape images with the three primary colors. R, G, B representation, can derive the various by either it can used for transformation process of both linear and nonlinear transformations.

\section{Linear transformation of images in yiq model}

YIQ is used for encoding the TV signal for American system in a color space systems. The values that are provided through different color components such as YUV, HSV, HIS, YUI. ${ }^{30}$

The color image was predicted towards the segmentation approaches by ad hoc networks. It can provide the strongly independent, results so we must improve the color images in vineyard fruit detections. These values are used to predicting the color images in the grape bunch.

\section{CONCLUSION}

The paper depicts the application of vineyard detection in different classifiers and it can be hybridized with other machine learning algorithms to achieve better accuracy. The performance analysis of the color fea- tures of grape bunches should be improved in future work The study has presented a comprehensive literature review of vineyard detection that focuses mainly on grape detections. As more research in the area, there is a need for a more recent literature review on the subject to and in the vineyard development field.

\section{REFERENCES}

1. Luo L, Tang Y, Zou X, Ye M, Feng W, Li G. Vision-based extraction of spatial information in grape clusters for harvesting robots. Biosystems Engineering. 2016;151:90-104.

2. Gongal A, Amatya S, Karkee M, Zhang O, Lewis K. Sensors and systems for fruit detection and localization: A review. Computers and Electronics in Agriculture. 2015;116:8-19.

3. ZhuY, Cao Z, Lu H, LiY, Xiao Y. In-field automatic observation of wheat heading stage using computer vision. Biosystems Engineering. 2016;143:28-41.

4. Jiménez AR, Ceres $R$, Pons JL. A vision system based on a laser rangefinder applied to robotic fruit harvesting. Machine Vision and Applications. 2000;11(6):321-9.

5. Grossetete M, Berthoumieu Y Da Costa JP Germain C Lavialle O, Grenier G. Early estimation of vineyard yield: site specific counting of berries by using a smartphone. In International Conference of Agricultural Engineering-CIGRAgEng. 2012.

6. Nuske S, Achar S, Bates T, Narasimhan S, Singh S. Yield estimation in vineyards by visual grape detection. In Intelligent Robots and Systems (IROS), IEEE/RSJ International Conference on 2011;2352-8. IEEE.

7. Zhang Y, Wu L. Classification of fruits using computer vision and a multiclass support vector machine. Sensors. 2012;12(9):12489-505

8. Zhao Y, Gong L, Zhou B, Huang Y, Liu C. Detecting tomatoes in greenhouse scenes by combining AdaBoost classifier and colour analysis. Biosystems Engineering. 2016;148:127-37.

9. Berenstein R, Shahar OB, Shapiro A, Edan Y. Grape clusters and foliage detection algorithms for autonomous selective vineyard sprayer. Intelligent Service Robotics. 2010;3(4):233-43.

10. Font D, Pallejà T, Tresanchez M, Teixidó M, Martinez D, Moreno J, et al. Counting red grapes in vineyards by detecting specular spherical reflection peaks in RGB images obtained at night with artificial illumination. Computers and electronics in agriculture. 2014;108:105-11.

11. Reis MJ, Morais R, Peres E, Pereira C, Contente O, Soares S, et al. Automatic detection of bunches of grapes in natural environment from color images. Journal of Applied Logic. 2012;10(4):285-90.

12. Cui Y, Fu L, Gejima Y, Su S, Wang B, Zhang F. Kiwifruit recognition at night using artificial lighting based on machine vision. In 2014 Montreal, Quebec Canada, 2014;1. American Society of Agricultural and Biological Engineers.

13. Font D, Tresanchez M, Martínez D, Moreno J, Clotet E, Palacín J. Vineyard yield estimation based on the analysis of high resolution images obtained with artificial illumination at night, Sensors. 2015;15(4):8284-301.

14. Cheng HD, Jiang $X H$, Sun $Y$, Wang J. Color image segmentation: advances and prospects. Pattern recognition. 2001;34(12):2259-81.

15. Tian LF, Slaughter DC. Environmentally adaptive segmentation algorithm for outdoor image segmentation. Computers and electronics in agriculture. 1998;21(3):153-68.

16. Smart RE. Principles of grapevine canopy microclimate manipulation with implications for yield and quality. A review. American Journal of Enology and Viticulture. 1985;36(3):230-9.

17. Teixidó $M$, Font $D$, Pallejà T, Tresanchez $M$, Nogués $M$, Palacín J. Definition of linear color models in the RGB vector color space to detect red peaches in orchard images taken under Natural illumination. Sensors. 2012;12(6):7701-18.

18. Liu S, Whitty M. Automatic grape bunch detection in vineyards with an SVM classifier. Journal of Applied Logic. 2015;13(4):643-53.

19. Nuske S, Achar S, Bates T, Narasimhan S, Singh S. Yield estimation in vineyards by visual grape detection. In Intelligent Robots and Systems (IROS), IEEE/RS J International Conference on. 2011;2352-8. IEEE.

20. Diago MP, Correa C, Millán B, Barreiro P, Valero C, Tardaguila J. Grapevine yield and leaf area estimation using supervised classification methodology on RGB images taken under field conditions. Sensors. 2012;12(12):16988-7006.

21. Wei $X$, Jia $K$, Lan J, Li $Y$, Zeng $Y$, Wang $C$. Automatic method of fruit object extraction under complex agricultural background for vision system of fruit picking robot. Optik-International Journal for Light and Electron Optics. 2014;125(19):5684-9.

22. Yamamoto K, Guo W, Yoshioka Y, Ninomiya S. On plant detection of intact tomato fruits using image analysis and machine learning methods. Sensors. 2014;14(7):12191-206

23. Zhao Y, Gong L, Huang Y, Liu C. Robust tomato recognition for robotic harvesting using feature images fusion. Sensors. 2016;16(2):173.

24. Zhang CX, Zhang JS, Zhang GY. An efficient modified boosting method for solving classification problems. Journal of Computational and Applied Mathematics. 2008;214(2):381-92.

25. Kong KK, Hong KS. Design of coupled strong classifiers in Ada Boost frame- 
work and its application to pedestrian detection. Pattern Recognition Letters. 2015;68:63-9.

26. Kicherer A, Herzog K, Pflanz M, Wieland M, Rüger P, Kecke S, et al. An automated field phenotyping pipeline for application in grapevine research. Sensors. 2015;15(3):4823-36.

27. Kotsiantis, Sotiris BI. Zaharakis, and P. Pintelas. "Supervised machine learning: A review of classification techniques." (2007): 3-24.
28. Freund $Y$, Schapire RE. Experiments with a new boosting algorithm. In Icml. 1996;96:148-56

29. Diago MP, Correa C, Millán B, Barreiro P, Valero C, Tardaguila J. Grapevine yield and leaf area estimation using supervised classification methodology on RGB images taken under field conditions. Sensors. 2012;12(12):16988-7006.

30. Si Y, Liu G, Feng J. Location of apples in trees using stereoscopic vision. Computers and Electronics in Agriculture. 2015;112:68-74.

Cite this article: Mary MVA, Sekar KR, Manikandan R. Improvement of Immune System Analyzed through Various Cluster Techniques - A Survey. Pharmacog J. 2018;10(2):256-9. 